\title{
Reticular basement membrane in asthma and COPD: Similar thickness, yet different composition
}

\author{
Jeroen JW Liesker' \\ Nick H Ten Hacken' \\ Mieke Zeinstra-Smith² \\ Steven R Rutgers' \\ Dirkje S Postma' \\ Wim Timens ${ }^{2}$ \\ 'Department of Pulmonology; \\ 2Department of Pathology, University \\ Medical Center Groningen, University \\ of Groningen, Groningen, \\ The Netherlands
}

\begin{abstract}
Background: Reticular basement membrane (RBM) thickening has been variably associated with asthma and chronic obstructive pulmonary disease (COPD). Even if RBM thickness is similar in both diseases, its composition might still differ.

Objective: To assess whether RBM thickness and composition differ between asthma and COPD. Methods: We investigated 24 allergic asthmatics (forced expiratory volume in one second $\left[\mathrm{FEV}_{1}\right] 92 \%$ predicted), and 17 nonallergic COPD patients $\left(\mathrm{FEV}_{1} 60 \%\right.$ predicted), and for each group a control group of similar age and smoking habits (12 and 10 persons, respectively). Snapfrozen sections of bronchial biopsies were stained with hematoxylin/eosin and for collagen I, III, $\mathrm{IV}, \mathrm{V}$, laminin and tenascin. RBM thickening was assessed by digital image analysis. Relative staining intensity of each matrix component was determined.

Results: Mean (SD) RBM thickness was not significantly different between asthma and COPD 5.5 (1.3) vs 6.0 (1.8) $\mu \mathrm{m}$, but significantly larger than in their healthy counterparts, ie, 4.7 (0.9) and 4.8 (1.2) $\mu \mathrm{m}$, respectively. Collagen I and laminin stained significantly stronger in asthma than in COPD. Tenascin stained stronger in asthma than in healthy controls of similar age, and stronger in COPD controls than in asthma controls $(\mathrm{p}<0.05)$.

Conclusion: RBM thickening occurs both in asthma and COPD. We provide supportive evidence that its composition differs in asthma and COPD.

Keywords: reticular basement membrane thickness, reticular basement membrane composition, asthma, biopsy, COPD, remodeling
\end{abstract}

\section{Introduction}

One of the hallmarks of airway remodeling is a thickened airway reticular basement membrane $(\mathrm{RBM})$ as a result of extracellular matrix deposition directly apposing the lamina reticularis. ${ }^{1,2}$ In an earlier study, Kasahara and colleagues compared the thickness of the RBM (biopsies) and of the whole airway wall thickness as assessed by high-resolution computed tomography (HRCT) in patients with mild-to-moderate asthma and healthy controls matched for age and sex. ${ }^{3}$ They showed that RBM thickness in asthmatic patients was larger than that of healthy controls and that it correlated strongly with whole airway wall thickness and forced expiratory volume in one second $\left(\mathrm{FEV}_{1}\right)$. Furthermore, James and colleagues showed similar findings comparing the reticular basement membrane thickness of central airways assessed by endobronchial biopsy and airway wall dimensions in cartilaginous airways in surgical specimens. ${ }^{4}$ Although disputed by others, ${ }^{5}$ these findings support earlier studies suggesting that RBM thickness may be used as an index of remodeling of the airway wall in asthma.

In contrast to asthma, the literature is not unanimous whether airway RBM is thickened in patients with chronic obstructive pulmonary disease (COPD). Some studies find a normal RBM, ${ }^{6-8}$ while others report it to be thickened,,${ }^{9-13}$ but generally 
to a smaller extent than in asthma. ${ }^{9,10,12,14,15}$ The differences in observations may be due to the fact that some studies have included only a few COPD patients ${ }^{7}$ or COPD patients with only mild ${ }^{10}$ or more severe ${ }^{9}$ airway obstruction. In addition, some studies included healthy controls who were either never smokers ${ }^{11}$ or smokers not being matched for pack-years smoking. ${ }^{8}$ Airway inflammation is of a chronic nature in both asthma and COPD, likely resulting in a long-lasting increase of fibrogenic growth factors like transforming growth factor$\beta$ in both asthma and COPD, ${ }^{10,16}$ vascular endothelial growth factor in asthma, ${ }^{17}$ and epidermal growth factors in COPD. ${ }^{10}$ Thus it might be anticipated that the RBM is thickened in both asthma and COPD.

Collagen IV, laminin, and proteoglycans are the major components of the true basement membrane. Beneath this thin layer, collagen I, III, V, and tenascin amongst others form the lamina reticularis, which is thickened in asthma patients. Both layers are strongly connected by strands of collagen VII. Laminin and tenascin have binding sites for the other matrix proteins, as well as for mesenchymal and epithelial cells. Together with proteoglycans, these matrix proteins form a firm and complex network that regulates processes like cell-migration and cell-adhesion.

We hypothesized that extracellular matrix deposition, resulting in $\mathrm{RBM}$ thickening, occurs in both asthma and COPD. Since the inflammatory profile differs between asthma and COPD, we anticipated that the extracellular matrix composition of RBM differs in terms of the relative contribution of each ECM component. We analyzed bronchial biopsies of patients with asthma and COPD with respect to the relationship between RBM thickness and composition on one hand, and $\mathrm{FEV}_{1}$, bronchial hyperresponsiveness on the other hand. Because the RBM thickness and composition may change during aging, we compared each group with age- and pack-years smoking-matched healthy controls.

\section{Methods}

\section{Subjects}

Subjects were recruited from our outpatient clinic of the University Medical Center Groningen or by advertisements in local newspapers. The local medical ethics committee approved the protocols and all subjects gave their written informed consent.

\section{Asthma patients and healthy controls}

Twenty-four subjects with mild-to-moderate severe asthma, 18-45 years old, were included. Their results of inflammatory composition of airway wall biopsies have been reported previously. ${ }^{18}$ Asthmatics were selected on: history consistent with asthma; positive intracutaneous tests against house dust mite or two other aeroallergens (18 common aeroallergens of ALK, Groningen, the Netherlands), $\mathrm{FEV}_{1}>60 \%$ predicted; provocative concentration of methacholine causing a $20 \%$ fall in $\mathrm{FEV}_{1}\left(\mathrm{PC}_{20}\right.$ methacholine $) \leq 9.8 \mathrm{mg} / \mathrm{ml}$; and provocative concentration of adenosine-5-monophosphate (AMP) causing a $20 \%$ fall in $\mathrm{FEV}_{1}\left(\mathrm{PC}_{20} \mathrm{AMP}\right) \leq 80 \mathrm{mg} / \mathrm{ml}$. Twelve healthy volunteers of similar age were selected on: no history of lung disease; $\mathrm{FEV}_{1}>85 \%$ predicted, no atopy and no airways hyperresponsiveness to methacholine or AMP. Asthmatic and healthy subjects who had been smoking in the past two years were excluded.

\section{COPD patients and healthy controls}

Seventeen COPD subjects over 45 years of age were included, according to American Thoracic Society (ATS) criteria. ${ }^{19}$ All subjects had a negative history of atopy, negative skin tests to 18 common aeroallergens (ALK, Groningen, the Netherlands) and negative specific IgE for 11 common aeroallergens (phadiatop ${ }^{\circledR}$ ). Subjects with COPD had an $\mathrm{FEV}_{1}$ and $\mathrm{FEV}_{1} /$ vital capacity $(\mathrm{VC})<$ predicted value -1.64 residual standard deviations ${ }^{20}$ and an increase in $\mathrm{FEV}_{1}<10 \%$ predicted after inhalation of $1 \mathrm{mg}$ terbutaline per Turbuhaler ${ }^{\circledR}$. Three subjects were never-smokers, all other participants were ex-smokers, ie they quitted smoking at least one year before the start of the study. Ten healthy ex-smoking volunteers were included without a history of pulmonary disease, with normal lung function, and similar age and pack-years smoking.

All patients stopped inhaled corticosteroids at least four weeks prior to bronchoscopy. Exclusion criteria were treatment with oral prednisolone and/or antibiotics, or a respiratory infection within four weeks prior to the study. Because of safety reasons, all patients had an $\mathrm{FEV}_{1}>1.5 \mathrm{~L}$.

\section{Lung function}

$\mathrm{FEV}_{1}$ was performed according to standardized guidelines of the European Respiratory Society. ${ }^{20}$ Reversibility was tested 30 minutes after inhalation with $400 \mu \mathrm{g}$ salbutamol in asthma and with $1 \mathrm{mg}$ terbutaline in COPD. Subjects were not allowed to use short-acting bronchodilators within 12 hours or long-acting bronchodilators within 24 hours.

\section{Bronchoscopy and processing of biopsies}

Bronchoscopy was performed using an Olympus B1 IT10 flexible fiberoptic bronchoscope (Olympus Optical, Tokyo, Japan) according to American Thoracic Society (ATS) criteria. ${ }^{21}$ 
At least five biopsies were taken from the subsegmental carinas from the left or right lower lobe using a fenestrated cup forceps (Olympus BF-21-C, Tokyo Japan).

Biopsies were mounted in Tissue Tek ${ }^{\circledR}$ embedding compound and snap-frozen by immersion in isopentane $\left(-80^{\circ} \mathrm{C}\right)$. Frozen biopsies were cut in serial sections at $4-\mu \mathrm{m}$ thickness. Selection of morphologically optimal tissue was based on every twentieth section, stained with Mayer's hematoxylin and eosin. Slides were stored at $-20^{\circ} \mathrm{C}$.

\section{Measuring of reticular basement membrane thickness}

Sections from all biopsies from each patient were stained with Mayer's hematoxylin and eosin. The morphologically most optimal section was selected by including sections with optimal integrity of the RBM and excluding tangentially cut tissue. RBM thickness was assessed by digital analysis (Leica Quantimed, Zeist, The Netherlands). Sections were coded and measured in random order at a magnification of 400x. One technician (MZ) performed all measurements. Fifteen measurements were performed over a cumulative length of basement membrane of $750 \mu \mathrm{m}$ in each section. These measurements were equally distributed over the total length of the basement membrane. For each measurement, an area of the RBM was marked over a length of $50 \mu \mathrm{m}$. Thereafter, the exact surface area of basement membrane and apposed matrix was assessed. Dividing the surface (calculated by the computer) by its exact length resulted in the mean RBM thickness of the marked surface area. The mean RBM thickness of an individual section was the mean of 15 measurements.

\section{Immunostaining}

Immunohistochemical staining was performed on $4 \mu \mathrm{m}$ sections of snap-frozen biopsies, dried at room temperature and fixed for 10 minutes in acetone. Slides were rinsed three times in phosphate-buffered saline (PBS) for five minutes between each step. Sections were stained for collagen I (goat polyclonal antibody; Southern Biotechnology Associates, Birmingham, AL, USA, see Figure 3), collagen III (mouse monoclonal antibody; Heyl, Berlin, Germany), collagen IV (rabbit polyclonal antibody; Cappel/ICN, Aurora, OH, USA), collagen V (goat polyclonal antibody; Southern Biotechnology Associates, Birmingham, AL, USA), laminin (rabbit polyclonal antibody; Telios, San Diego, CA, USA), and tenascin (mouse monoclonal antibody; Telios). Subsequently, the sections were put in PBS with $0.075 \% \mathrm{H}_{2} \mathrm{O}_{2}$ for 30 minutes to block endogenous peroxidase activity. Finally, sections were treated with species-specific peroxidaseconjugated immunoglobulins (DAKO; Glostrup, Denmark) for 30 minutes and peroxidase staining was performed using 3-amino-ethylcarbazole (AEC; Sigma Chemical Company, St. Louis, MO, USA) as a substrate, providing a reddishbrown reaction product.

\section{Examination of (reticular) basement membrane components}

Semi-quantitative examination was performed by one investigator (MZ) in a blinded fashion using a light microscope at 100x magnification. Before semi-quantitative scoring of individual sections, all sections immuno-stained for a specific antigen were examined in order to have an impression of the range of immunopositivity. Thereafter, sections were scored on a three-point scale for staining intensity of the reticular basement membrane (1, low; 2, moderate; 3, high intensity). Intra-observer agreements (kappa, standard error) for collagen I, III, IV, V, laminin, and tenascin were: $0.74(0.14), 0.81(0.13), 0.80(0.13), 0.51$ $(0.19), 0.84(0.11)$, and $0.73(0.14)$, respectively.

\section{Data analysis}

Analyses were performed with the SPSS (version 10.0; SPSS Inc., Chicago, IL, USA) software package. Normality of distribution was assessed with the Kolmogorov-Smirnov test. Differences in RBM thickness were evaluated by ANOVA followed by t-test, differences in staining activity of matrix components by Mann-Whitney U Test. Correlations between RBM thickness and $\mathrm{FEV}_{1}$ were evaluated by Pearson tests, correlations between RBM thickness and composition on the one hand, and $\mathrm{PC}_{20} \mathrm{AMP}$ on the other hand with Spearman tests. P-values $\leq 0.05$ were considered to be significant.

\section{Results \\ Subjects}

Clinical characteristics of the patients with asthma and COPD, and their healthy controls are listed in Table 1. After analyzing RBM thickness, two subjects with asthma and one subject with COPD did not yield sufficient tissue to evaluate the RBM composition.

\section{Reticular basement membrane thickness}

Values of the RBM thickness showed a normal distribution in all groups. The variability in RBM thickness was not significantly different between asthma and COPD. Mean (standard deviation) RBM thickness in asthma and COPD was significantly greater than that of healthy controls of 
Table I Baseline characteristics

\begin{tabular}{|c|c|c|c|c|}
\hline & Asthma & Asthma controls & COPD & COPD controls \\
\hline Numbers & 24 & 12 & 17 & 10 \\
\hline Gender, M/F & $15 / 9$ & $4 / 8$ & $13 / 4$ & $8 / 2$ \\
\hline Age, years & $31(9.1)$ & $31(8.2)$ & $62(7.5)$ & $59(7.8)$ \\
\hline $\mathrm{FEV}_{1}, \%$ pred & $92(12.6)$ & $105(13.8)$ & $60(13.0)$ & $105(10.5)$ \\
\hline Reversibility, FEV , \% pred & II.5 (9.7) & $1.6(2.2)$ & $5.4(2.5)$ & $3.0(3.2)$ \\
\hline Pack years & - & - & $27.8(21.0)$ & $25.8(15.1)$ \\
\hline Current smoking & 0 & 0 & 0 & 0 \\
\hline $\mathrm{PC}_{20} \mathrm{AMP}, \mathrm{mg} / \mathrm{ml}$ & $2.5(0.18-22.8)$ & $>80$ & $|2|(\mid-267)$ & $>80$ \\
\hline $\mathrm{PC}_{20}$ methacholine, $\mathrm{mg} / \mathrm{ml}$ & $0.42(0.8-6.6 \mathrm{I})$ & $>9.8$ & Not done & Not done \\
\hline
\end{tabular}

Note: Data are presented in mean (standard deviation) or median (range).

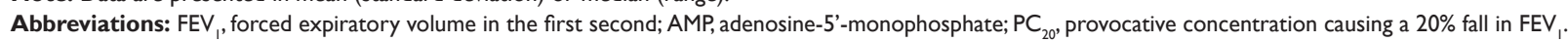

similar age: 5.5 (1.3) $\mu \mathrm{m}$ vs 4.7 (0.9) $\mu \mathrm{m}$ in asthma, and 6.0 (1.8) vs $4.8(1.2) \mu \mathrm{m}$ in COPD (Figure 1).

\section{Composition of the reticular basement membrane}

Assessment of the RBM staining with collagen I, III, IV, V, tenascin, and laminin showed that collagen I (Figure 3) and laminin staining of the RBM were significantly more intense in asthma than in COPD $(\mathrm{p}<0.05$, Figure 2$)$. In contrast, collagen IV showed a trend towards more intense staining in COPD than in asthma ( $p=0.084$, Figure 2). Tenascin staining of the RBM was significantly more intense in asthmatics than in healthy controls ( $p<0.05$, Figure 2 ), but not significantly different from COPD. Tenascin staining was significantly more intense in the COPD than in the asthma control group (Figure 2). No further significant differences were found between the four study groups.

\section{Correlations of basement membrane and clinical characteristics}

There were no significant correlations between RBM thickness or extracellular matrix components (Collagen I, III, $\mathrm{IV}, \mathrm{V}$, tenascin, and laminin) on one hand and $\mathrm{FEV}_{1} \%$ pred, $\mathrm{PC}_{20} \mathrm{AMP}$, and $\mathrm{PC}_{20}$ methacholine (in asthma only) on the other hand, neither in asthmatics, nor in COPD patients, nor in healthy subjects.

\section{Discussion}

This study shows that the reticular airway basement membrane is thickened both in patients with mild-to-moderate asthma and COPD, as compared to healthy subjects of similar age and smoking history. An interesting observation is that the extracellular matrix composition of the reticular basement membrane differs between asthma and COPD, the latter containing significantly less collagen I and laminin. The reticular basement membrane thickness and its extracellular matrix composition did not significantly correlate with parameters of lung function.

To our knowledge this is the first study comparing RBM thickness in asthma and COPD patients and their healthy controls of similar age and smoking history. An important finding of this study is that the airway RBM in central airway biopsies is thickened with a similar magnitude in asthma and COPD. Other reports give contrasting results. ${ }^{7,9,10,12,14,15,22}$ There are several explanations for this difference. Two studies compared asthma patients with a mixed group of COPD and chronic bronchitis patients (thus individuals with and without

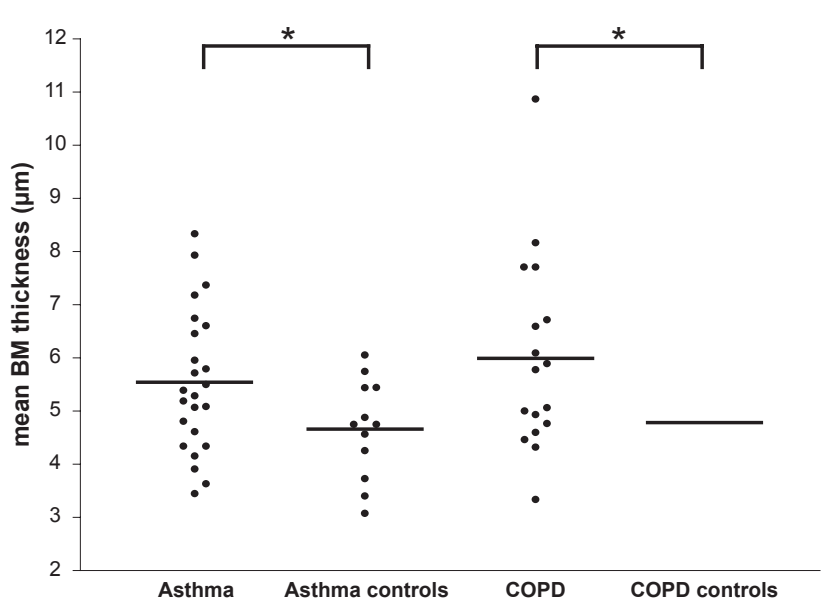

Figure I Mean basement membrane thickness for asthma, COPD, and healthy controls of similar age. Reticular basement membrane thickness in central airway wall biopsies from 24 patients with asthma, 12 age-matched healthy controls of asthma, 17 patients with COPD and 10 age- and pack year-matched healthy controls of COPD.

Notes: Solid lines are means; ${ }^{*} \mathrm{p}<0.05$.

Abbreviation: COPD, chronic obstructive pulmonary disease. 
airway obstruction) finding a thicker RBM in asthma than in the mixed group. ${ }^{10,22}$ Two other studies reported a thicker RBM in asthma than in COPD, ${ }^{7,9}$ however these studies did not report their data in a quantitative way. Our study has the advantage that a more homogeneous group of COPD patients was selected, and additionally that RBM was measured using a computerized morphometry system, providing quantitative and non-selective data. In line with our results, Bourdin and colleagues demonstrated RBM thickness of a comparable magnitude as our findings in (mild) asthma and COPD and reported that this is not a useful histological marker to differentiate between these diseases. ${ }^{13,23}$ In the latter study, a measurement method similar to ours was used which has been validated by Wilson and colleagues. ${ }^{24} \mathrm{We}$ also demonstrate that the mean SBM thickness in asthma and COPD patients is larger than in healthy controls, though with a large overlap. This overlap is in line with earlier studies, despite the use of different methods and use of healthy controls with different age and/or smoking status. ${ }^{1,5,7,9-13,15,17,22,24-44}$ Taken together, we conclude that an increased RBM thickness indicates the presence of asthma or COPD, irrespective of age and that it is not possible to differentiate mild-to-moderate asthma from COPD purely based on RBM thickness. In addition, a normal RBM thickness does not exclude obstructive airway disease.

One could postulate that the age difference between our asthma and COPD patients, or asthma severity is responsible for the lack of difference in RBM thickness. Earlier studies have shown that RBM thickness is not associated with asthma duration, ${ }^{25,26,45-47}$ age, ${ }^{20,27}$ or asthma severity. ${ }^{12,26,27}$ Notwithstanding this, Bourdin and colleagues recently provided evidence that particularly patients with severe asthma have a larger thickness of the RBM than asthmatics with mild disease. The latter two groups had similar duration of disease, confirming that it is not duration but rather severity of disease that affects RBM thickness. Our data for the first time show that age and aging may not affect RBM thickness,
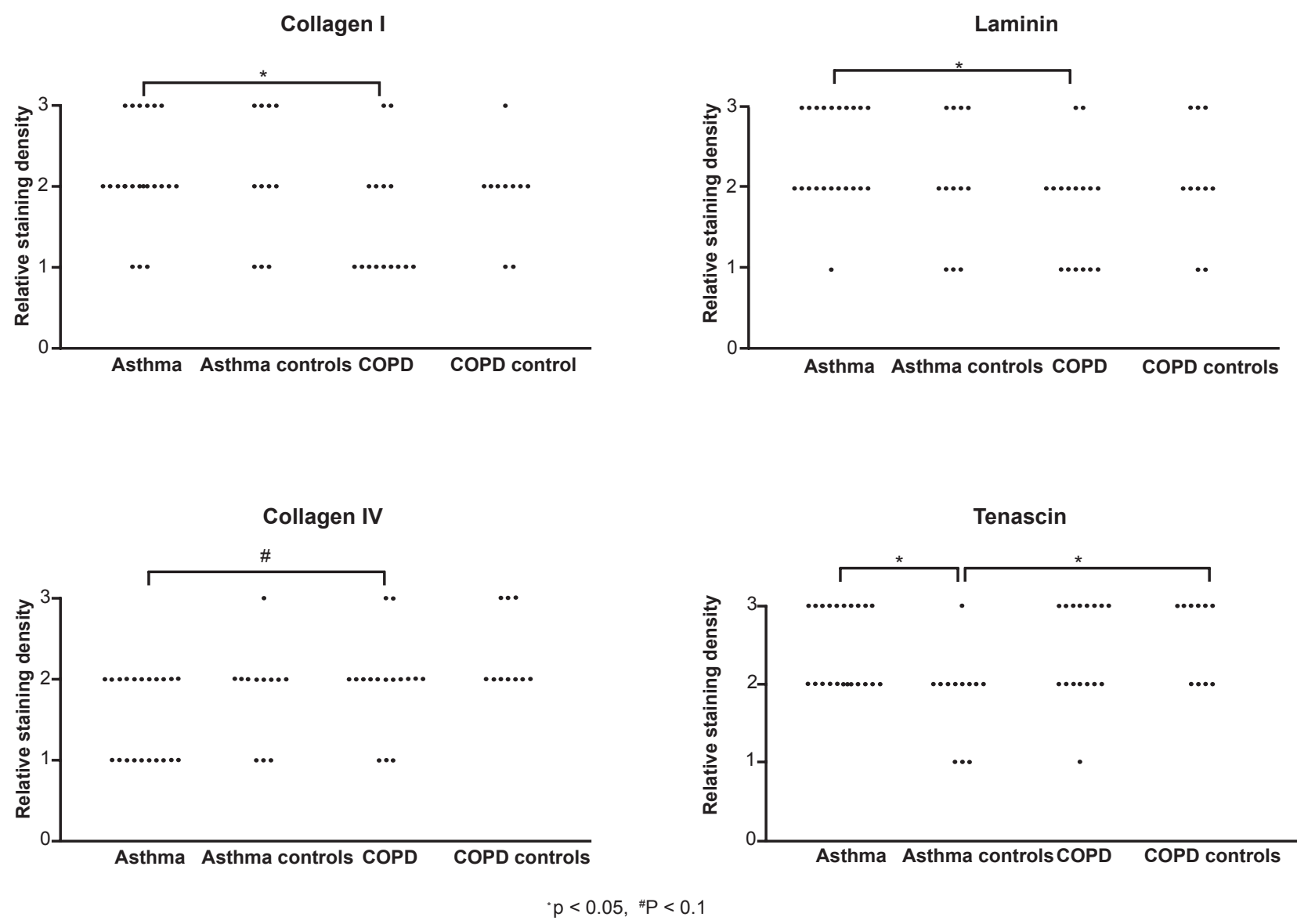

Figure 2 Reticular basement membrane composition. Relative staining density of collagen I, laminin, collagen IV, and tenascin in the reticular basement membrane of airway wall biopsies in asthma and COPD patients, and their healthy matched control subject. Results of collagen III and V are not shown as there were no significant differences between any of the groups.

Notes: ${ }^{*} \mathrm{p}<0.05,{ }^{*} \mathrm{p}=0.08$

Abbreviation: COPD, chronic obstructive pulmonary disease. 
A)

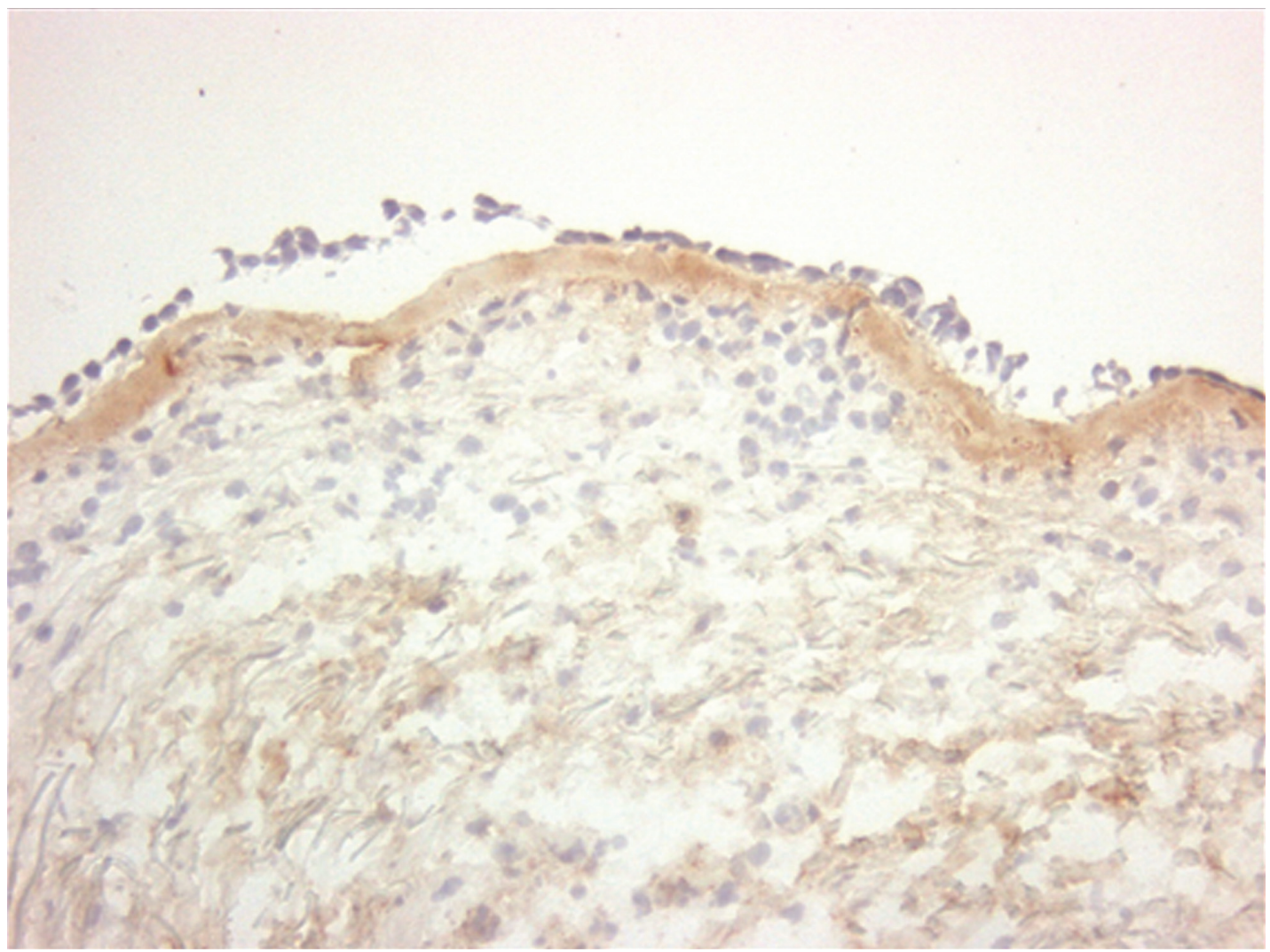

B)

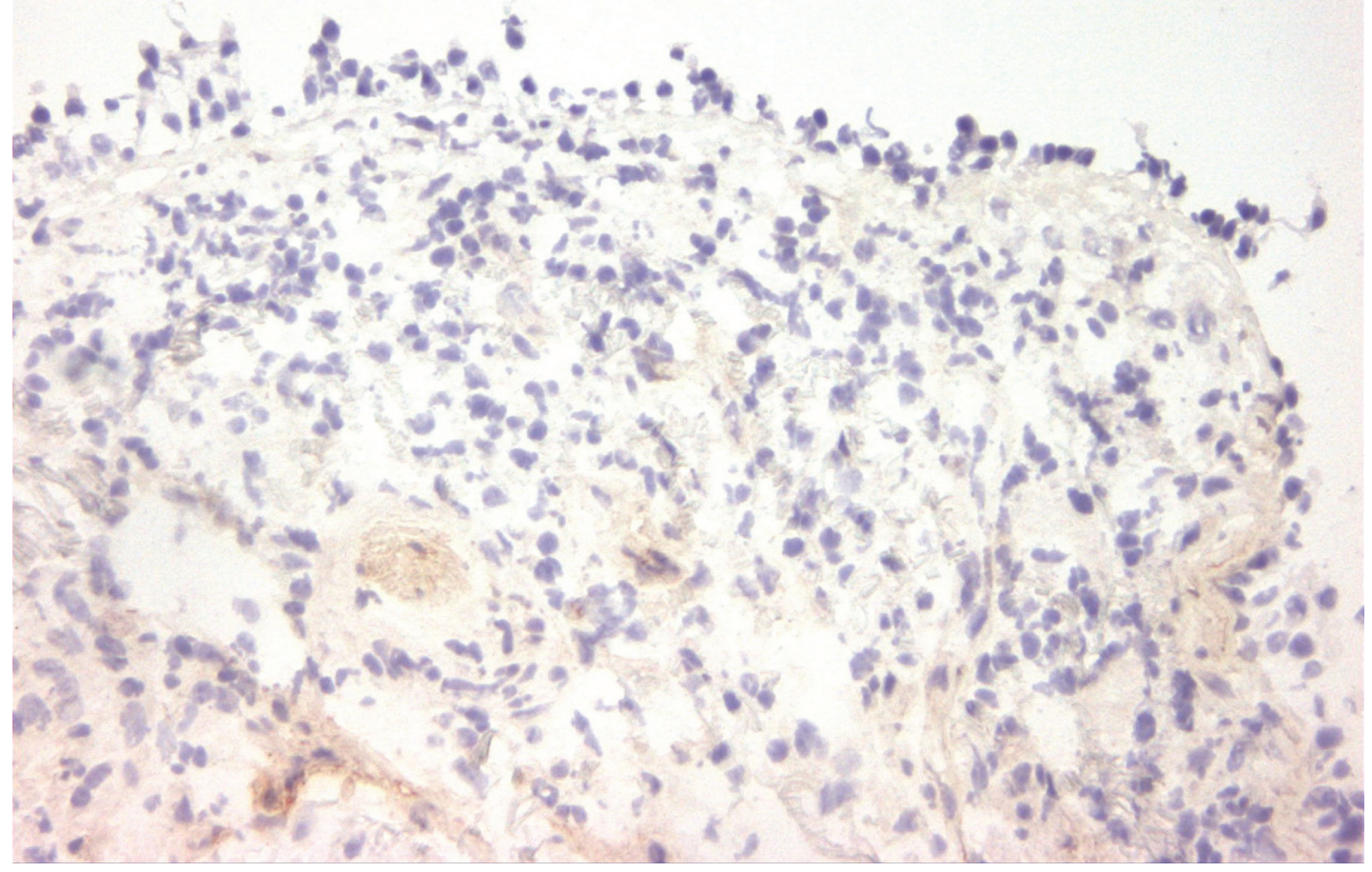

Figure 3 Collagen I staining. Immunohistochemical staining of bronchial biopsies for collagen I (immunoperoxidase, original magnification $\times 200$ ) showed, a uniform red-brown-stained band beneath the epithelial layer in asthma ( $\mathbf{A}$ left panel). In contrast to asthma, this is significantly less intense in COPD ( $\mathrm{P}<0.05, \mathbf{B}$ right panel). Abbreviation: COPD, chronic obstructive pulmonary disease. 
but they do affect the composition of the RBM, since tenascin was significantly more present in COPD controls than in the asthma control group, which by design of the study was younger in age. Alternatively, this may be the result of long-time smoke exposure, because COPD controls were ex-smokers and asthma controls were never-smokers.

The mean RBM thickness in our study is somewhat smaller in both asthma and COPD than in most other studies. This can be the result of using snap-frozen biopsies instead of embedding biopsies in resin or paraffin, or differences in fixation techniques. The manner of measuring RBM thickness is to our opinion decisive for the observed differences. First, we used area measurements, which Bourdin and colleagues showed to result in lower values than when using line measurements. ${ }^{13}$ By using area measurements, we evaluated a significantly larger part of the RBM to assess thickness; for example we used 15 areas of $50 \mu \mathrm{m}$ length while Sullivan used 40 line measurements. ${ }^{48}$ Second, we measured completely random, large areas of RBM, while line measurements are more prone to selection bias. Thus our method has advantage over previous publications in that we used a more unbiased measuring method. ${ }^{12,14,15}$

This study is the first to demonstrate that the extracellular matrix composition of the RBM differs between asthma and COPD, yet with large overlap in staining pattern. Previous studies have used staining of different collagens, but did not asses its further composition. ${ }^{24,30,33-36,40}$ The observed differences between asthma and COPD in the extracellular matrix composition may be due to different types of irritation, epithelial damage, epithelial repair, and underlying submucosal airway inflammation, both in a quantitative and qualitative way. Collagen IV is a component of the "true" basement membrane which is not thickened in asthma. Compared to asthma, collagen IV showed a trend for a higher staining intensity in COPD.

The above differences in composition underline differences in pathophysiology of both diseases. Tenascin stained stronger in asthma than in healthy controls of similar age and also stronger in (older) COPD controls than in (younger) asthma controls. Kranenburg and colleagues compared the staining intensity of the RBM in patients with COPD and healthy subjects in surgical resection specimens using a similar scoring system as in our study. ${ }^{49}$ Their results showed enhanced expression of total collagen, collagen I, and -III, but not of collagen IV, fibronectin, and laminin. Differences with our findings are likely caused by the use of surgical resection specimens whereas we investigated large airway bronchoscopic biopsies.
A thickened RBM and a change in its composition are both features of airway remodelling, which is supposed to contribute to airflow limitation in asthma and COPD. This study shows no significant correlations of RBM thickness and composition with $\mathrm{FEV}_{1} \%$ predicted, in line with some $^{25,30,47}$ but not all studies. ${ }^{13,18,27,34}$ Obviously, different results may be explained by differences in study populations and morphometric methods. Furthermore, we could not demonstrate a significant correlation between $\mathrm{PC}_{20} \mathrm{AMP}$ and $\mathrm{RBM}$ thickness. With respect to $\mathrm{PC}_{20}$ methacholine several other studies have shown a negative correlation with RBM thickness in asthma. ${ }^{25,29,34,50}$ This would suggest that $\mathrm{PC}_{20}$ methacholine is more closely related to markers of airway remodeling than $\mathrm{PC}_{20} \mathrm{AMP}$, yet our data did not confirm this. Whether a thickened basement membrane is beneficial or harmful for the natural course of asthma or COPD, eg, by protecting against allergen or smoke exposure, is not supported by long-term follow-up studies.

In summary, this study shows that the reticular basement membrane is thickened in both asthma and COPD, yet has a different composition. More studies are needed to elucidate the exact relationship between the process of ongoing airway inflammation and airway remodeling in these diseases.

\section{Acknowledgments}

Jeroen Liesker has received an unrestricted research salary grant of AstraZeneca, Benelux. Studies in asthma and COPD were supported by the Dutch Asthma Foundation, Stichting Astma Bestrijding, and AstraZeneca, Benelux.

\section{Disclosure}

None of the authors has any financial interest, or any actual or potential conflict of interest in the subjects discussed in this manuscript.

\section{References}

1. Dunnill MS, Massarella GR, Anderson JA. A comparison of the quantitative anatomy of the bronchi in normal subjects, in status asthmaticus, in chronic bronchitis, and in emphysema. Thorax. 1969;24(2):176-179.

2. Bousquet J, Jeffery PK, Busse WW, Johnson M, Vignola AM. Asthma. From bronchoconstriction to airways inflammation and remodeling. Am J Respir Crit Care Med. 2000;161(5):1720-1745.

3. Kasahara K, Shiba K, Ozawa T, Okuda K, Adachi M. Correlation between the bronchial subepithelial layer and whole airway wall thickness in patients with asthma. Thorax. 2002;57(3):242-246.

4. James AL, Maxwell PS, Pearce-Pinto G, Elliot JG, Carroll NG. The relationship of reticular basement membrane thickness to airway wall remodeling in asthma. Am J Respir Crit Care Med. 2002; 166(12 Pt 1):1590-1595.

5. Jeffery PK, Wardlaw AJ, Nelson FC, Collins JV, Kay AB. Bronchial biopsies in asthma. An ultrastructural, quantitative study and correlation with hyperreactivity. Am Rev Respir Dis. 1989;140(6):1745-1753. 
6. Chanez P, Vignola AM, O'Shaugnessy T, et al. Corticosteroid reversibility in COPD is related to features of asthma. Am J Respir Crit Care Med. 1997;155(5):1529-1534.

7. Ollerenshaw SL, Woolcock AJ. Characteristics of the inflammation in biopsies from large airways of subjects with asthma and subjects with chronic airflow limitation. Am Rev Respir Dis. 1992;145(4 Pt 1):922-927.

8. Vachier I, Vignola AM, Chiappara G, et al. Inflammatory features of nasal mucosa in smokers with and without COPD. Thorax. 2004;59(4):303-307.

9. Lacoste JY, Bousquet J, Chanez P, Van Vyve T, Simony-Lafontaine J, Lequeu N, et al. Eosinophilic and neutrophilic inflammation in asthma, chronic bronchitis, and chronic obstructive pulmonary disease. J Allergy Clin Immunol. 1993;92(4):537-548.

10. Vignola AM, Chanez P, Chiappara G, et al. Transforming growth factorbeta expression in mucosal biopsies in asthma and chronic bronchitis. Am J Respir Crit Care Med. 1997;156(2 Pt 1):591-599.

11. Pesci A, Majori M, Cuomo A, et al. Neutrophils infiltrating bronchial epithelium in chronic obstructive pulmonary disease. Respir Med. 1998;92(6):863-870.

12. Benayoun L, Druilhe A, Dombret MC, Aubier M, Pretolani M. Airway structural alterations selectively associated with severe asthma. Am J Respir Crit Care Med. 2003;167(10):1360-1368.

13. Bourdin A, Neveu D, Vachier I, Paganin F, Godard P, Chanez P. Specificity of basement membrane thickening in severe asthma. J Allergy Clin Immunol. 2007;119(6):1367-1374.

14. Fabbri LM, Romagnoli M, Corbetta L, et al. Differences in airway inflammation in patients with fixed airflow obstruction due to asthma or chronic obstructive pulmonary disease. Am J Respir Crit Care Med. 2003;167(3):418-424.

15. Milanese M, Crimi E, Scordamaglia A, et al. On the functional consequences of bronchial basement membrane thickening. $J$ Appl Physiol. 2001;91(3):1035-1040.

16. Takizawa $\mathrm{H}$, Tanaka $\mathrm{M}$, Takami $\mathrm{K}$, et al. Increased expression of transforming growth factor-betal in small airway epithelium from tobacco smokers and patients with chronic obstructive pulmonary disease (COPD). Am J Respir Crit Care Med. 2001;163(6):1476-1483.

17. Chetta A, Zanini A, Foresi A, et al. Vascular endothelial growth factor up-regulation and bronchial wall remodelling in asthma. Clin Exp Allergy. 2005;35(11):1437-1442.

18. Ten Hacken NH, Timens W, Smith M, Drok G, Kraan J, Postma DS. Increased peak expiratory flow variation in asthma: severe persistent increase but not nocturnal worsening of airway inflammation. Eur Respir J. 1998;12(3):546-550.

19. Standards for the diagnosis and care of patients with chronic obstructive pulmonary disease. American Thoracic Society. Am J Respir Crit Care Med. 1995;152(5 Pt 2):S77-S121.

20. Quanjer PH, Tammeling GJ, Cotes JE, Pedersen OF, Peslin R, Yernault JC. Lung volumes and forced ventilatory flows. Work Group on Standardization of Respiratory Function Tests. European Community for Coal and Steel. Official position of the European Respiratory Society. Rev Mal Respir. 1994;11(Suppl 3):5-40.

21. Summary and recommendations of a workshop on the investigative use of fiberoptic bronchoscopy and bronchoalveolar lavage in asthmatics. Am Rev Respir Dis. 1985;132(1):180-182.

22. O'Shaughnessy TC, Ansari TW, Barnes NC, Jeffery PK. Reticular basement membrane thickness in moderately severe asthma and smokers with and without airflow obstruction. Am J Respir Crit Care Med. 1996;153:A879.

23. Bourdin A, Serre I, Flamme H, et al. Can endobronchial biopsy analysis be recommended to discriminate between asthma and COPD in routine practice? Thorax. 2004;59(6):488-493.

24. Wilson JW, Li X. The measurement of reticular basement membrane and submucosal collagen in the asthmatic airway. Clin Exp Allergy. 1997;27(4):363-371.

25. Hoshino M, Nakamura Y, Sim JJ. Expression of growth factors and remodelling of the airway wall in bronchial asthma. Thorax. 1998;53(1):21-27.
26. Payne DN, Rogers AV, Adelroth E, et al. Early thickening of the reticular basement membrane in children with difficult asthma. Am J Respir Crit Care Med. 2003;167(1):78-82.

27. Shiba K, Kasahara K, Nakajima H, Adachi M. Structural changes of the airway wall impair respiratory function, even in mild asthma. Chest. 2002;122(5):1622-1626.

28. Boulet LP, Laviolette M, Turcotte H, et al. Bronchial subepithelial fibrosis correlates with airway responsiveness to methacholine. Chest. 1997;112:45-52.

29. Cho SH, Seo JY, Choi DC, et al. Pathological changes according to the severity of asthma. Clin Exp Allergy. 1996;26:1210-1219.

30. Chu HW, Halliday JL, Martin RJ, Leung DY, Szefler SJ, Wenzel SE. Collagen deposition in large airways may not differentiate severe asthma from milder forms of the disease. Am J Respir Crit Care Med. 1998;158(6):1936-1944.

31. Roche WR, Beasley R, Williams JH, Holgate ST. Subepithelial fibrosis in the bronchi of asthmatics. Lancet. 1989;1(8637):520-524.

32. Jeffery PK, Godfrey RW, Adelroth E, Nelson F, Rogers A, Johansson SA. Effects of treatment on airway inflammation and thickening of basement membrane reticular collagen in asthma. A quantitative light and electron microscopic study. Am Rev Respir Dis. 1992;145(4 Pt 1):890-899.

33. Wenzel SE, Schwartz LB, Langmack EL, et al. Evidence that severe asthma can be divided pathologically into two inflammatory subtypes with distinct physiologic and clinical characteristics. Am J Respir Crit Care Med. 1999;160(3):1001-1008.

34. Hoshino M, Nakamura Y, Sim J, Shimojo J, Isogai S. Bronchial subepithelial fibrosis and expression of matrix metalloproteinase-9 in asthmatic airway inflammation. J Allergy Clin Immunol. 1998;102(5): 783-788.

35. Laitinen LA, Laitinen A, Altraja A, et al. Bronchial biopsy findings in intermittent or "early" asthma. J Allergy Clin Immunol. 1996;98(5 Pt 2): S3-S6.

36. Chakir J, Laviolette M, Boutet M, Laliberte R, Dube J, Boulet LP. Lower airways remodeling in nonasthmatic subjects with allergic rhinitis. $L a b$ Invest. 1996;75(5):735-744.

37. Brewster CE, Howarth PH, Djukanovic R, Wilson J, Holgate ST, Roche WR. Myofibroblasts and subepithelial fibrosis in bronchial asthma. Am J Respir Cell Mol Biol. 1990;3(5):507-511

38. Chanez P, Vignola AM, Vic P, et al. Comparison between nasal and bronchial inflammation in asthmatic and control subjects. Am J Respir Crit Care Med. 1999;159(2):588-595.

39. Braunstahl GJ, Fokkens WJ, Overbeek SE, Kleinjan A, Hoogsteden $\mathrm{HC}$, Prins JB. Mucosal and systemic inflammatory changes in allergic rhinitis and asthma: a comparison between upper and lower airways. Clin Exp Allergy. 2003;33(5):579-587.

40. Ward C, Johns DP, Bish R, et al. Reduced airway distensibility, fixed airflow limitation, and airway wall remodeling in asthma. Am J Respir Crit Care Med. 2001;164(9):1718-1721.

41. Brightling CE, Symon FA, Birring SS, Bradding P, Wardlaw AJ, Pavord ID. Comparison of airway immunopathology of eosinophilic bronchitis and asthma. Thorax. 2003;58(6):528-532.

42. Barbato A, Turato G, Baraldo S, et al. Epithelial damage and angiogenesis in the airways of children with asthma. Am J Respir Crit Care Med. 2006;174(9):975-981.

43. Chetta A, Zanini A, Foresi A, et al. Vascular component of airway remodeling in asthma is reduced by high dose of fluticasone. Am J Respir Crit Care Med. 2003;167(5):751-757.

44. Siddiqui S, Mistry V, Doe C, et al. Airway hyperresponsiveness is dissociated from airway wall structural remodeling. J Allergy Clin Immunol. 2008;122(2):335-341.

45. Bai TR, Cooper J, Koelmeyer T, Pare PD, Weir TD. The effect of age and duration of disease on airway structure in fatal asthma. Am J Respir Crit Care Med. 2000;162(2 Pt 1):663-669.

46. Boulet LP, Turcotte H, Laviolette M, et al. Airway hyperresponsiveness, inflammation, and subepithelial collagen deposition in recently diagnosed versus long-standing mild asthma. Influence of inhaled corticosteroids. Am J Respir Crit Care Med. 2000;162(4 Pt 1):1308-1313. 
47. Tillie-Leblond I, de BJ, Jaubert F, Wallaert B, Scheinmann P, Gosset P. Airway remodeling is correlated with obstruction in children with severe asthma. Allergy. 2008;63(5):533-541.

48. Sullivan P, Stephens D, Ansari T, Costello J, Jeffery P. Variation in the measurements of basement membrane thickness and inflammatory cell number in bronchial biopsies. Eur Respir J. 1998;12(4):811-815.
49. Kranenburg AR, Willems-Widyastuti A, Moori WJ, et al. Enhanced bronchial expression of extracellular matrix proteins in chronic obstructive pulmonary disease. Am J Clin Pathol. 2006;126(5):725-735.

50. Ward C, Pais M, Bish R, et al. Airway inflammation, basement membrane thickening and bronchial hyperresponsiveness in asthma. Thorax. 2002;57(4):309-316. 
